\title{
Equipment and Methods for Rapid Analysis of PWO Full-Sized Scintillation Crystal Radiation Hardness During Mass Production
}

\author{
Gleb Drobychev, Alexander Annenkov, Andrei Fedorov, Mikhali Korzhik, Paul Lecoq, Andrei Lopatik, \\ Oleg Missevitch, Jean-Pierre Peigneux, Alexander Singovski, and Roman Zouevski
}

\begin{abstract}
The mass production of lead tungstate crystals (PWO) for the Compact Muon Solenoid (CMS) Project at CERN began at the Bogoroditsk Techno-Chemical Plant (BTCP, Tula Region, Russia) in 2000. Mass production technology, developed in recent years, is based on a set of methods and instrumentation for crystal growth and machining, as well as quality control and certification of crystals. One of the most crucial categories of tolerances is the radiation hardness of crystals. Control of the PWO radiation hardness during the mass production phase requires a reliable, easy-to-use measuring tool with high productivity. A semiautomatic spectrometric setup for PWO radiation hardness monitoring was developed and tested at CERN. After final crosschecks, the setup was put into operation at BTCP.
\end{abstract}

Index Terms-Certification, electromagnetic calorimeters, radiation hardness, scintillation crystals.

\section{REQUIREMENTS FOR PWO CRYSTAL RADIATION HARDNESS}

$\mathbf{T}$ HE requirements on radiation tolerance, set by the specification for the preproduction phase, are intended to provide the best possible compromise between electromagnetic calorimeter barrel performance and crystal production yield [1]. As is stated in the specification, the required level of PWO radiation hardness is defined by three parameters:

1) induced absorption $\mu$ of fully saturated crystal, laterally irradiated by ${ }^{60} \mathrm{Co}$ with a total absorbed dose $>500 \mathrm{~Gy}$ at a dose rate $>100 \mathrm{~Gy} / \mathrm{h}$ at $420 \mathrm{~nm}$ is $\leq 1.5 \mathrm{~m}^{-1}$ (measured within $40 \mathrm{~min}$ after irradiation, $\mathrm{T}=18^{\circ} \mathrm{C}$ );

2) a light yield loss $<6 \%$ after frontal ${ }^{60} \mathrm{Co}$ irradiation for a total absorbed dose of $2 \mathrm{~Gy}$ and a dose rate of $0.15 \mathrm{~Gy} / \mathrm{h}$ corresponding to the large hadron collider (LHC) irradiation environment (measured periodically during irradiation, $\mathrm{T}=18^{\circ} \mathrm{C}$ );

3) no recovery time constant shorter than $1 \mathrm{~h}$.

Manuscript received October 15, 2000; revised February 3, 2001 and March 30,2001 . This work was supported in part by the International Scientific and Technical Center (Moscow) under Project 354B.

G. Drobychev, A. Fedorov, M. Korzhik, A. Lopatik, O. Missevitch, and R. Zouevski are with the Institute for Nuclear Problems, Minsk 220050, Belarus (e-mail: Gleb.Drobychev@cern.ch; fyod@inp.minsk.by; Mikhail.Korjik@cern.ch; lopatik@inp.minsk.by; misov@inp.minsk.by; Roman.Zouevski@cern.ch).

A. Annenkov is with the Bogoroditsk Technical Chemical Plant, Tula Region, Bogoroditsk, Russia (e-mail: annenkov@ btcp.tula.net).

P. Lecoq is with CERN, Geneva 23, Switzerland (e-mail: Paul.Lecoq@cern.ch).

J.-P. Peigneux and A. Singovski are with LAPP, Annecy-le-Vieux F-74941, France. (e-mail: Peigneux@lapp.in2p3.fr; Alexander.Singovski@cern.ch).

Publisher Item Identifier S 0018-9499(01)07391-9.
During the optimization of the lead tungstate production technology, numerous irradiation tests were performed to measure PWO crystal behavior in the irradiation environment similar to one of LHC and to tune the specification of the crystals. Besides the specification tuning, these tests provided valuable feedback to crystal technology development. Although various irradiation methods gave results differing by a factor of about two [2] and the information return to the producer was rather slow, in the end the technology of radiation-hard PWO crystal production was achieved [3]-[5]. After this, the stabilization of the level of technology reached becomes crucial, requiring development of adequate methods and equipment for fast control of crystal quality during mass production. From this point of view, the crystal producer has all the needed instrumentation for crystal geometry and optical property control, namely, an automatic crystal control system (ACCOS), which includes a three-dimensional machine [6]. However, the organization of a reliable control of radiation hardness met some obstacles. First of all, such measurements require a long time. Taking into account the total quantity of crystals under production, it is impossible to measure the radiation hardness of each crystal at an accelerator facility.

\section{Semiautomatic Spectrometric SetuP For PWO RADIATION HARDNESS CONTROL}

To provide the producer with a device for crystal irradiation and radiation hardness measurements, a semiautomatic spectrometric setup was developed.

The method of radiation hardness estimation is based on our previous research on the radiation damage mechanism in PWO crystals consistent with results of other research groups. It was found that radiation centers of PWO crystals are not affected by irradiation in a wide region of doses. The detected loss of light yield is caused by the appearance of radiation-induced optical absorption [5], [7], [8]. That is why we proposed to estimate PWO radiation hardness through measurements of the optical transmission change under irradiation. Appropriate research was carried out in order to find a reliable correlation between values of light yield loss and radiation-induced absorption at a specified wavelength [9].

The setup includes a light-insulated transportable container, an inner container with optical windows designed for simultaneous irradiation of four crystals, and a four-channel optical spectrometer for measurements of longitudinal induced absorption at three different wavelengths. The setup is intended for 


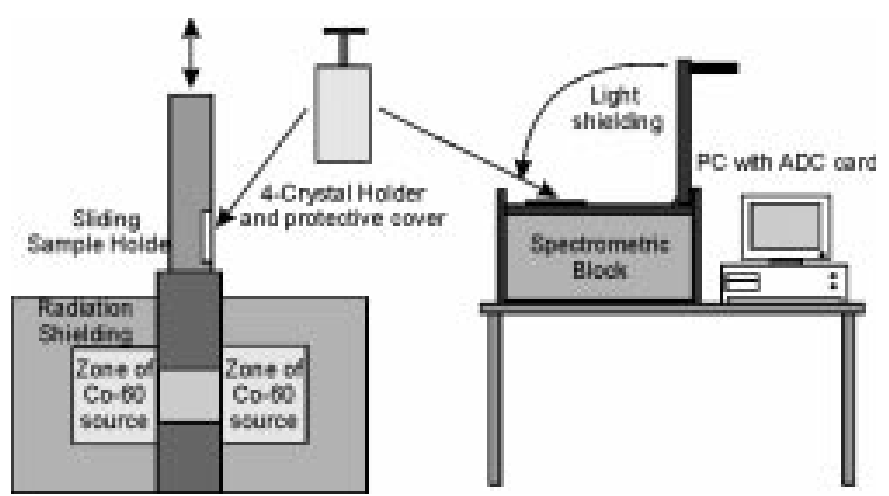

Fig. 1. Spectrometric setup at irradiation facility.

use at the irradiation facility located at $70 \mathrm{~km}$ from Bogoroditsk Techno-Chemical Plant (BTCP). There is a ${ }^{60} \mathrm{Co}$ source of round geometry installed in a radiation-protecting well. The cylindrical inner container placed in the well is irradiated laterally from all sides with a dose rate of about $100 \mathrm{~Gy} / \mathrm{h}$. The measurement procedure is illustrated in Fig. 1. It starts with the initial transmission measurement of crystals. Then, the inner container has to be removed from the spectrometer and placed in the well. After irradiation of the crystals, the container has to be installed again in the spectrometer to measure the transmission of damaged crystals. Since the transmission of crystal can be measured with good precision in several minutes, the recovery time constant can also be estimated through repeated transmission measurements at fixed time intervals.

\section{THE OPTICAL SPECTROMETER}

The spectrometer operation is based on the measurement of the PWO longitudinal optical transmission change after irradiation at three wavelengths.

In the final design, the following wavelengths were chosen: $630 \mathrm{~nm}$ (red), $525 \mathrm{~nm}$ (green), and $460 \mathrm{~nm}$ (blue). Consequently, the spectrometric setup was called "RGB system."

The choice of wavelengths was determined for the following reasons: $420 \mathrm{~nm}$ corresponds to the maximum of PWO emission; $525 \mathrm{~nm}$ corresponds to the maximum of sensitivity of avalanche photodiodes that will be used in the CMS electromagnetic calorimeter; and $630 \mathrm{~nm}$ is a reference point where radiation-induced absorption is negligible. The curve of radiation-induced absorption is nearly flat in $400-480 \mathrm{~nm}$ region. That allows us to use very recent superbright "blue" diodes, which are now available with wavelengths shorter than $470 \mathrm{~nm}$.

The version of developed software that is currently installed at the BTCP irradiation facility calculates directly the induced crystal absorption instead of the relative loss of the light-emitting diode (LED) signal (light yield loss).

The block diagram of the spectrometer is represented in Fig. 2.

Three LEDs emit in turn short (tens of nanoseconds), stable light pulses. Light from each LED is mixed by four diffusion cavities and illuminates the PWO scintillators in the longitudinal direction (see Fig. 3). After passing through the crystal and diffusing on the receiving reflector, the light hits the PIN

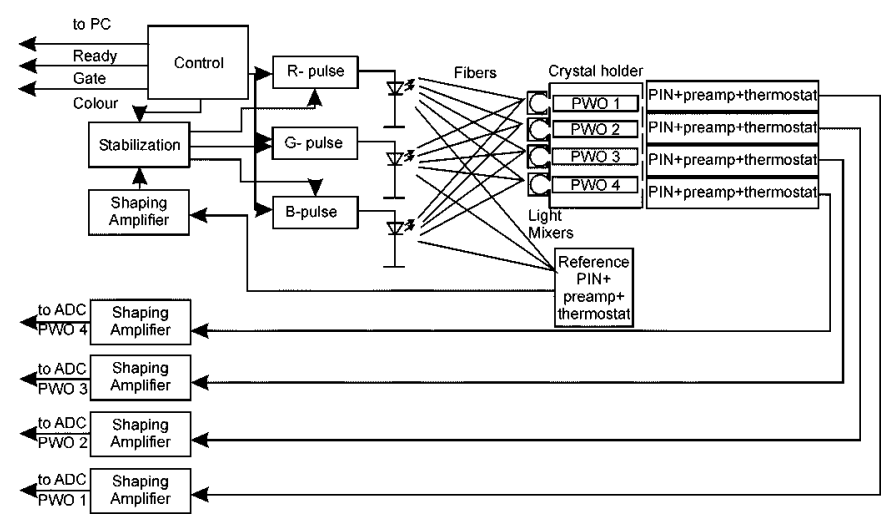

Fig. 2. Optical spectrometer block diagram.

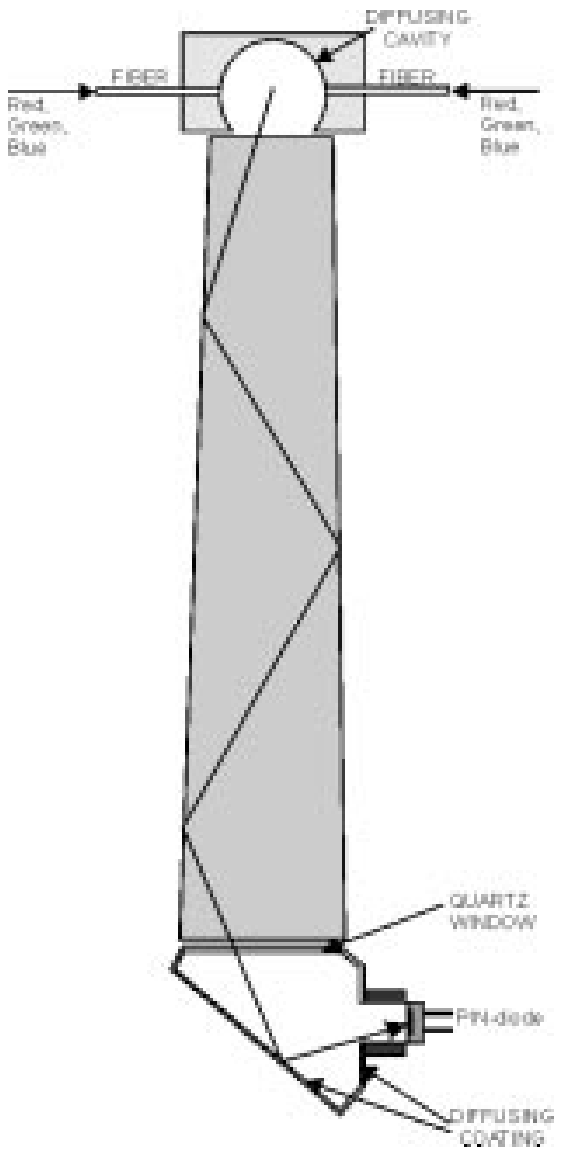

Fig. 3. Light mixer and photodetector geometry of a single channel.

photodiodes. The PIN diode and charge-sensitive preamplifier are placed in a thermostatic box that ensures their long-term stability at the level of $10^{-4}{ }^{\circ} \mathrm{C}$ determined by the precision of the thermostabilizer. The preamplifier signals are shaped in duration and amplitude by a precision-shaping amplifier and sent to a custom-made PC-based ADC card.

Some part of the light from the LEDs is directed to a reference channel, which consists of a thermostabilized PIN diode, a charge-sensitive preamplifier, and a shaping amplifier. The signal from the shaping amplifier is compared with the reference voltage level, and the resulting "error" signal operates the LED driving circuit. This principle of automated control of light pulse intensity is similar to that described in detail in [10]. 
TABLE I

BASIC CHARACTERISTICS OF THE RGB SYSTEM

\begin{tabular}{|c|c|c|c|c|c|c|c|c|}
\hline $\begin{array}{l}\text { Number of } \\
\text { simuitaneously } \\
\text { measured } \\
\text { crystals }\end{array}$ & Emitted $v$ & elength of 1 & Ds, nm & $\begin{array}{l}\text { Number of } \\
\text { photons in } \\
\text { pulse per } \\
\text { channel, } \\
\text { min }\end{array}$ & $\begin{array}{l}\text { Average } \\
\text { time of } \\
\text { transmission } \\
\text { measurement, } \\
\text { min }\end{array}$ & $\begin{array}{l}\text { Overall } \\
\text { long-term } \\
\text { stability of } \\
\text { system, } \%\end{array}$ & $\begin{array}{l}\text { Time of } \\
\text { warming- } \\
\text { up and self } \\
\text { test, min }\end{array}$ & $\begin{array}{l}\text { Time of } \\
\text { continuous } \\
\text { work }\end{array}$ \\
\hline 4 & $\begin{array}{c}460 \\
\text { ("Blue") }\end{array}$ & $\begin{array}{c}525 \\
\text { ("Green") }\end{array}$ & $\begin{array}{c}630 \\
\left({ }^{(*} \operatorname{Red}^{* \prime}\right)\end{array}$ & 500000 & 5 & \pm 0.1 & 20 & not limited \\
\hline
\end{tabular}

The block "Control" synchronizes the operation of the spectrometer, also producing signals "Color," "Gate," and "Ready," which are necessary for ADC operation.

The operation of the device is completely automated. After switching on, an autocalibration is performed. It includes adjustment of photon number per channel and of amplification gain for each channel. This procedure is repeated twice immediately after switching on and after 20 min of warming up. The basic characteristics of the spectrometer are presented in Table I.

\section{RADIATION-HARDNESS TEST RESULTS}

Using the spectrometric setup described above, we performed a series of measurements at BTCP as well as at the General Irradiation Facility (GIF, CERN), the Geneva Hospital Cobalt source, and at the Minsk irradiation facility in order to find correlation between irradiation data obtained up to then for different irradiation conditions using different methods and instrumentation. Briefly, the main characteristics of these setups can be summarized as follows.

1) The GIF facility is equipped with a ${ }^{137} \mathrm{Cs} \gamma$-radiation source. This source provides lateral irradiation with an absorption dose rate of $0.15 \mathrm{~Gy} / \mathrm{h}$, which is close to the irradiation conditions of the CMS detector. Also, it is possible to use recurring crystal irradiation by electron beam. The GIF facility allows one to control the radiation damage by online measurement of the optical transmission loss as well as radiation damage kinetics of full-sized crystals with an accuracy of about $10 \%$.

2) The Minsk irradiation facility is equipped with a ${ }^{60} \mathrm{Co}$ source with an absorption dose rate of about $100 \mathrm{~Gy} / \mathrm{min}$. It allows the radiation damage to be checked by measurement of the induced optical absorption in small PWO samples at the saturation dose with an accuracy of about $5 \%$. The delay between irradiation and measurements is $40 \mathrm{~min}$.

3) The BTCP irradiation facility is equipped with a ${ }^{60} \mathrm{Co}$ source, which provides an absorption dose rate of about $100 \mathrm{~Gy} / \mathrm{h}$. The RGB semiautomatic setup allows one to estimate the radiation damage as well as the kinetics of the radiation damage recovery by measurement of the induced optical absorption of full-sized crystals at the saturation dose with an overall accuracy of measurement procedure of about $5 \%$. The delay between irradiation and measurements is $3 \mathrm{~min}$.

4) The Geneva hospital facility is equipped with a ${ }^{60} \mathrm{Co}$ source, which provides an absorption dose rate of about $250 \mathrm{~Gy} / \mathrm{h}$. It allows one to check the radiation damage by measurement of the induced optical absorption of

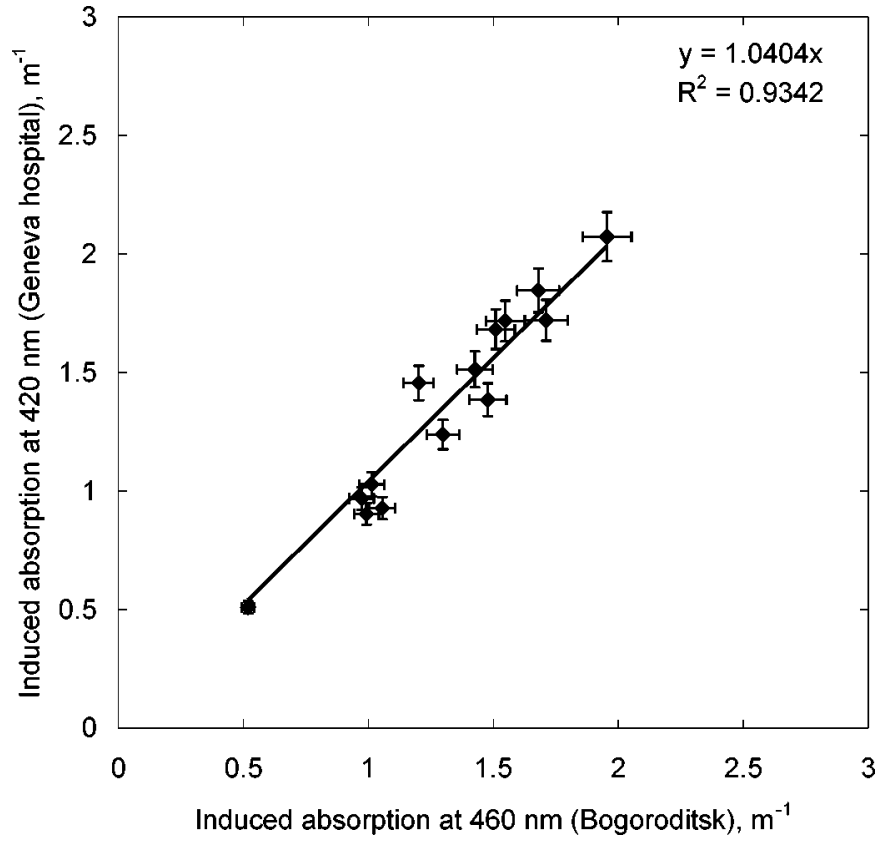

Fig. 4. Correlation between radiation-induced absorption measured at the Geneva Hospital and at the BTCP (RGB System). R-Pearson product moment correlation coefficient.

full-sized crystals at the saturation dose with an accuracy of about 5\%. The delay between irradiation and measurements is $40 \mathrm{~min}$.

We have accepted that the radiation damage in PWO crystals is the result of an equilibrium between processes of damage and recovery [11], [12]. In this case, the kinetics of radiation damage processes depends on absorbed dose and dose rate. Therefore, measurements are carried out, both at relatively low $(0.15 \mathrm{~Gy} / \mathrm{h})$ and at saturation $(>100 \mathrm{~Gy} / \mathrm{h})$ radiation dose rates.

Thus, these four irradiation facilities allowed us to make a complete study of the electromagnetic component of the PWO crystals radiation damage in the LHC irradiation environment.

Through a series of measurements, radiation hardness of the same crystals was evaluated. The accuracy of all instruments was evaluated through repeated measurements of the same samples. After each irradiation, crystals were annealed to ensure their recovery. These tests allowed us to find a correlation between the results of measurements obtained in different conditions.

Fig. 4 presents the correlation between values of radiation-induced absorption measured at the Geneva Hospital $\left(\mathrm{Co}^{60}\right.$ source, absorption dose rate of about $250 \mathrm{~Gy} / \mathrm{h}$, absorbed dose $500 \mathrm{~Gy}$, delay between irradiation and measurements of $\left.40 \mathrm{~min}, \mathrm{~T}=18^{\circ} \mathrm{C}\right)$ and at BTCP using RGB system $\left(\mathrm{Co}^{60}\right.$ 


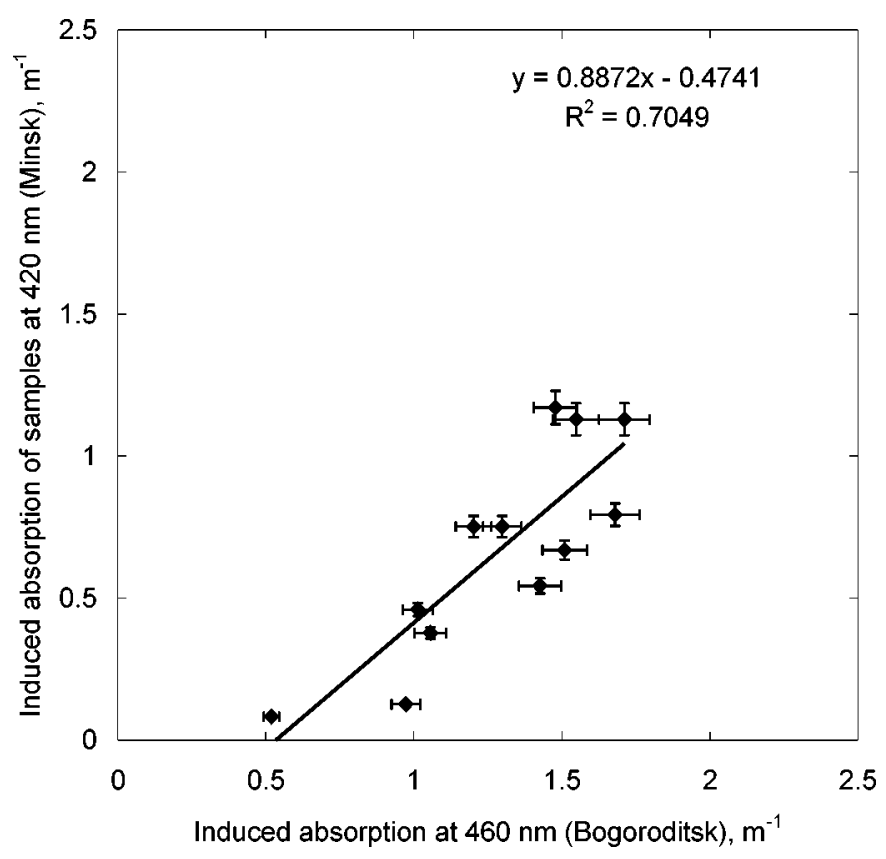

Fig. 5. Correlation between radiation-induced absorption of full-sized elements measured at BTCP (RGB System) and samples extracted from top parts of crystals (Minsk). R-Pearson product moment correlation coefficient.

source, absorption dose rate of about $100 \mathrm{~Gy} / \mathrm{h}$, absorbed dose $40 \mathrm{~Gy}$, delay between irradiation and measurements of $3 \mathrm{~min}$, $\mathrm{T}=18^{\circ} \mathrm{C}$ ). As one can see, a good correlation exists between measurements made with laboratory spectrometric equipment and the RGB system in case of irradiation with saturation doses.

Fig. 5 presents the correlation between radiation-induced absorption of full-sized elements measured at BTCP using RGB system (same conditions as quoted above) and samples extracted from the top parts of crystals measured at the Minsk irradiation facility $\left({ }^{60} \mathrm{Co}\right.$ source, absorption dose rate 100 Gy/min, absorbed dose $1000 \mathrm{~Gy}$, delay between irradiation and measurements of $40 \mathrm{~min}, \mathrm{~T}=18^{\circ} \mathrm{C}$ ). This correlation is worse than the previous one because of peculiarities in the PWO crystal growth processes. The presence of a constant term $(-0.4741)$ is due to a better radiation hardness of crystal material in the top part of the crystal ingot.

Fig. 6 shows the correlation between the radiation hardness of PWO crystals measured at the GIF facility $\left(\mathrm{Cs}^{137}\right.$ source, absorption dose rate of about $0.15 \mathrm{~Gy} / \mathrm{h}$, absorbed dose $6 \mathrm{~Gy}$, online measurements, $\mathrm{T}=18^{\circ} \mathrm{C}$ ) and at BTCP using RGB system (same conditions as quoted above). As can be seen, despite the limited accuracy and low statistics of the measurements available at the time of the GIF system startup, a correlation between values of radiation-induced absorption at saturation and at low doses was found. The presence of a constant term (0.0795) is due to better sensitivity of more sophisticated laboratory GIF equipment in comparison with one of the RGB system designed for rapid analysis at the mass production facility.

\section{CONCLusions}

Using the spectrometric setup described above, we performed a series of measurements at the Bogoroditsk and other irradia-

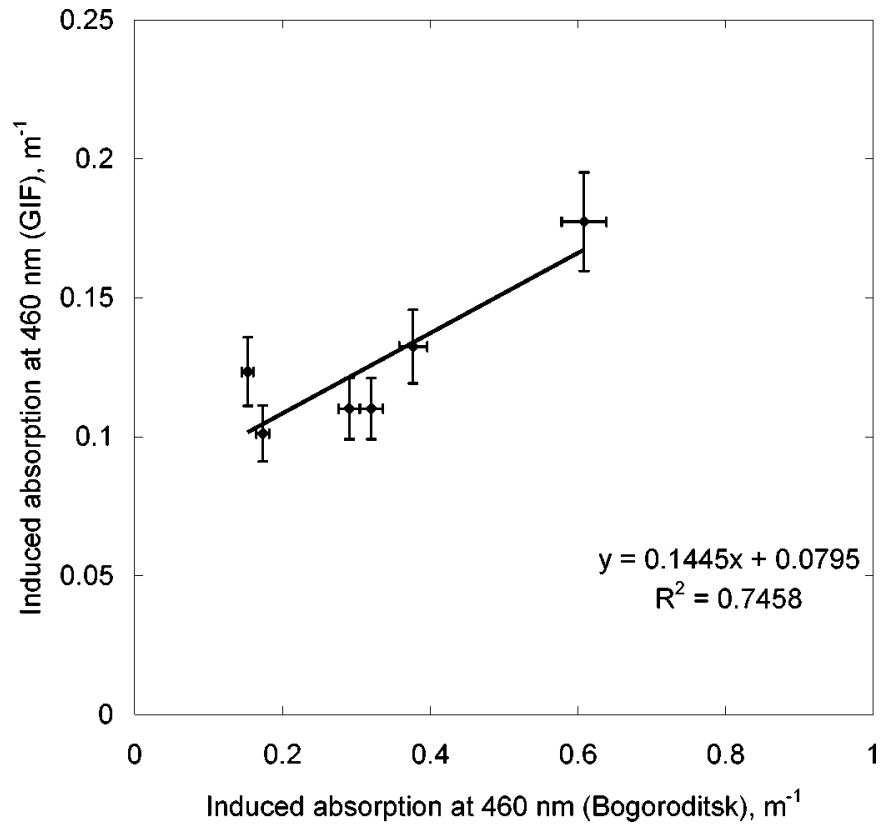

Fig. 6. Correlation between radiation-induced absorption of PWO crystals measured at the GIF facility (CERN) and at BTCP (RGB system). R-Pearson product moment correlation coefficient.

tion facilities in order to find a correlation between irradiation data obtained up to then with different methods and instrumentation. After transfer of the setup to BTCP, final crosschecks and calibrations were made at the producer irradiation facility during the year 2000.

The results presented exhibit a good linear correlation of the developed RGB setup with Geneva hospital facility, which was accepted as the standard for the CMS experiment. Consequently, the RGB setup was accepted as the principal system in CMS for radiation hardness control of mass-produced PWO crystals at the producer level. However, there is still poor correlation with results of the GIF facility. Taking into account that the GIF facility provides a unique possibility for online research of processes of radiation damage and recovery, additional efforts have to be made in order to increase the accuracy of measurements at the GIF facility and to confirm correlation with the results of the measurements of the RGB system.

As is shown in [13], by using the RGB system, it is possible to organize an effective method to control the crystal radiation hardness on a sampling basis. Analysis shows that the use of the methods and of the equipment developed so far allows us to minimize the quantity of crystals with bad radiation hardness in the CMS electromagnetic calorimeter and to reduce significantly the rejection of good crystals during the certification.

\section{ACKNOWLEDGMENT}

The authors are very grateful to Dr. J.-L.Faure of CERN for continuous encouragement for the PWO radiation hardness study.

They would also like to thank Dr. E. Auffray (CERN) and V. Ligun (BTCP), P. Ivankin (BTCP), and L. Matveev (BTCP) for their help during the preparation of the experiments. 


\section{REFERENCES}

[1] E. Auffray, M. Lebeau, P. Lecoq, and M. Schneegans, "Specification for lead tungstate crystals preproduction,", CMS Note 98/038, 1998.

[2] E. Auffray, P. Lecoq, P. Sempere-Roldan, J.-P. Peigneux , A. Singovski , and K. Deiters et al., "Comparison of different Irradiation procedures for recently optimized Russian $\mathrm{PbWO}_{4}$ crystals,", CMS Note 98/069, 1998.

[3] A. N. Annenkov, E. Auffray, A. E. Borisevich, G. Y. Drobychev, A. A. Fedorov, and O. V. Kondratiev et al., "Slow components and afterglow in PWO crystal scintillations," Nucl. Instrum. Meth., vol. A 403, pp. 302-312, 1998 .

[4] M. Böhm, A. E. Borisevich, G. Y. Drobychev, A. Hofstaetter, O. V. Kondratiev, and M. V. Korzhik et al., "Influence of Mo impurity on the spectroscopic and scintillation properties of $\mathrm{PbWO}_{4}$ crystals," Phys. Statist. Solidi, vol. A167, no. 1, pp. 243-252, May 1998.

[5] A. N. Annenkov, E. Auffray, R. Chipaux, G. Y. Drobychev, A. A. Fedorov, and M. Geleoc et al., "Systematic study of the $\mathrm{PbWO}_{4}$ crystal short - term instability under irradiation," Rad. Meas., vol. 29, no. 1, pp. 27-38, 1998.

[6] E. Auffray, G. Chevenier, R. Chipaux, G. Y. Drobychev, G. Dromby, and A. A. Fedorof et al., "Certifying procedures for lead tungstate crystal parameters during mass production for the CMS ECAL," in Conf. Rec. 1998 IEEE Nuclear Science Symp. Medical Imaging Conf., 1998.
[7] A. N. Annenkov, E. Auffray, M. V. Korzhik, P. Lecoq, and J.-P. Peigneux, "On the origin of the transmission damage in lead tungstate crystals under irradiation,” Phys. Statist. Solidi, vol. A170, pp. 47-62, 1998.

[8] R. Y. Zhu, D. A. Ma, H. B. Newman, C. L. Woody, J. A. Kierstead, and S. P. Stoll et al., "A study on the properties of lead tungstate crystals," Nucl. Instrum. Meth., vol. A376, pp. 319-334, 1996.

[9] A. N. Annenkov, E. Auffray, R. Chipaux, G. Y. Drobychev, A. A. Fedorov, and A. V. Singovski et al., "Control of lead tungstate crystals radiation hardness through optical transmission measurements," in Proc VII Int. Conf. Calorimetry in High Energy Physics, Tucson, AZ , 1997.

[10] A. Fedorov, M. Korzhik, A. Lopatik, and O. Missevitch, "LED ligh pulser for high precision monitoring of the scintillation calorimeter energy scale," Nucl. Instrum. Meth., vol. A413, pp. 352-356, 1998.

[11] A. N. Annenkov, E. Auffray, A. Fedorov, S. Gninenko, N. Golubev, and M. V. Korzhik et al., "Radiation damage kinetics in PWO crystals,", CMS Note 1997/008, 1997.

[12] R. Y. Zhu, "Precision crystal calorimetry in future high energy colliders," IEEE Trans. Nucl. Sci., vol. 44, no. 3, pp. 468-476, 1997.

[13] G. Drobychev, A. Annenkov, E. Auffray-Hillemans, A. Borisevitch, P. Lecoq, and V. Ligun et al., "Radiation hardness of mass produced PWO crystals," in Conf. Rec. 2000 IEEE Nuclear Science Symp. Medical Imaging, 2000. 